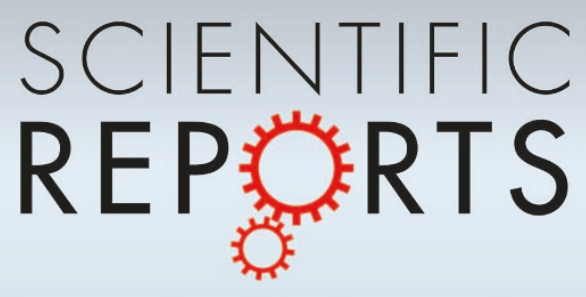

OPEN

SUBJECT AREAS:

ELECTRONIC PROPERTIES

AND DEVICES

ELECTRONIC PROPERTIES AND MATERIALS

ELECTRICAL AND ELECTRONIC ENGINEERING

GRAPHENE

Received

19 April 2013

Accepted

24 June 2013

Published

9 July 2013

Correspondence and requests for materials should be addressed to

D.V.V. (vyalikh@ physik.phy.tu-dresden.

de)

\section{Controlled assembly of graphene-capped nickel, cobalt and iron silicides}

\author{
O. Vilkov ${ }^{1,2}$, A. Fedorov ${ }^{1,3}$, D. Usachov' ${ }^{1}$, L. V. Yashina ${ }^{4}$, A. V. Generalov ${ }^{1,2}$, K. Borygina ${ }^{1}$, N. I. Verbitskiy ${ }^{4,5}$, \\ A. Grüneis ${ }^{3,5}$ \& D. V. Vyalikh ${ }^{1,2}$
}

${ }^{1}$ St. Petersburg State University, Ulyanovskaya str. 1, St. Petersburg 198504, Russia, ${ }^{2}$ Institute of Solid State Physics, Dresden University of Technology, Zellescher Weg 16, D-01062 Dresden, Germany, ${ }^{3}$ Leibniz-Institut für Festkörper- und Werkstoffforschung Dresden, Helmholtzstr. 20, P.O. Box 2701 16, D-0 1171 Dresden, Germany, ${ }^{4}$ M.V. Lomonosov Moscow State University, Leninskie Gory 1/3 119991 Moscow, Russia, ${ }^{5}$ Faculty of Physics, University of Vienna, Boltzmanngasse 5 A-1090 Vienna, Austria.

The unique properties of graphene have raised high expectations regarding its application in carbon-based nanoscale devices that could complement or replace traditional silicon technology. This gave rise to the vast amount of researches on how to fabricate high-quality graphene and graphene nanocomposites that is currently going on. Here we show that graphene can be successfully integrated with the established metal-silicide technology. Starting from thin monocrystalline films of nickel, cobalt and iron, we were able to form metal silicides of high quality with a variety of stoichiometries under a Chemical Vapor Deposition grown graphene layer. These graphene-capped silicides are reliably protected against oxidation and can cover a wide range of electronic materials/device applications. Most importantly, the coupling between the graphene layer and the silicides is rather weak and the properties of quasi-freestanding graphene are widely preserved.

or almost ten years, graphene, a two-dimensional crystal of carbon atoms packed in a honeycomb structure, has been in the focus of intensive research. Its remarkable and promising electronic properties have raised high expectations regarding its potential use in the next generation electronics of a post-silicon era ${ }^{1}$. However, a graphene-based technology first and foremost requires reliable and low-cost approaches to fabrication of high-quality and large-scale graphene layers on a variety of functional substrates. A major challenge is the integration of graphene into the current $\mathrm{Si}$-based technology, for which original solutions have become available recently ${ }^{2-6}$. One basic idea is to use silicon to create a semiconducting ${ }^{7-9}$ or an insulating ${ }^{10}$ buffer between $^{-}$ graphene and a metallic substrate, and thus to obtain structures that are suitable for electronic applications.

We propose to use monocrystalline films of nickel, cobalt and iron as the substrates, on top of which highquality graphene can be easily created using the Chemical Vapor Deposition (CVD) approach ${ }^{11-15}$. The silicides of these three metals in particular exhibit a variety of functional properties ${ }^{16,17}$ and are already extensively used. It is worth noting that metal silicides are generally currently employed for many applications including complementary metal oxide semiconductor (MOS) devices ${ }^{18,19}$, thin film coatings ${ }^{20}$, photovoltaics ${ }^{21,22}$, thermoelectrics $^{23}$, catalysts ${ }^{24}$ and biomolecule sensing ${ }^{25}$.

For instance, the use of $\mathrm{Ni}$ silicides as metal gate electrodes in complementary MOS devices has attracted particular attention ${ }^{26}$. Ni-rich silicides, like $\mathrm{Ni}_{3} \mathrm{Si}, \mathrm{Ni}_{2} \mathrm{Si}$ and $\mathrm{Ni}_{31} \mathrm{Si}_{12}$, can be used for p-MOS gates due to their higher work function, while $\mathrm{NiSi}, \mathrm{NiSi}_{2}$ and $\mathrm{Ni}_{3} \mathrm{Si}_{2}$ are attractive for $\mathrm{n}-\mathrm{MOS}$ applications ${ }^{27}$. $\mathrm{Ni}$ and Co silicides also exhibit intriguing electronic properties, especially in the form of nanowires or nanobelts ${ }^{28-31}$. The compound $\mathrm{CoSi}_{2}$, similarly to Ni silicides, is a material of choice to contact the source, drain and gate areas in complementary $\operatorname{MOS}^{18,19}$. The quite different electronic and magnetic properties of Fe-based silicides ${ }^{32,33}$ ensure huge potential of these materials for various applications, too ${ }^{34,35}$. For example, $\alpha-\mathrm{FeSi}_{2}$ exhibits metallic properties while $\beta$-FeSi $i_{2}$ is a narrow-gap semiconductor. Ferromagnetic $\mathrm{Fe}_{3} \mathrm{Si}$ layers can be used as a source for injection of spin-polarized electrons into a semiconductor ${ }^{36,37}$.

The aim of this work is to uncover the basic properties of the graphene/silicide interfaces. Here, we demonstrate that intercalation of silicon atoms underneath the graphene top layer and a series of annealing cycles allow one to create a variety of metal silicides with different properties, all nicely covered and protected by graphene. The graphene layer itself barely interacts with the silicides underneath and shows quasi-freestanding character. We anticipate a wide range of opportunities where the properties of such silicides, the graphene layer on top of the semiconducting silicides or of both combined could be utilized. 


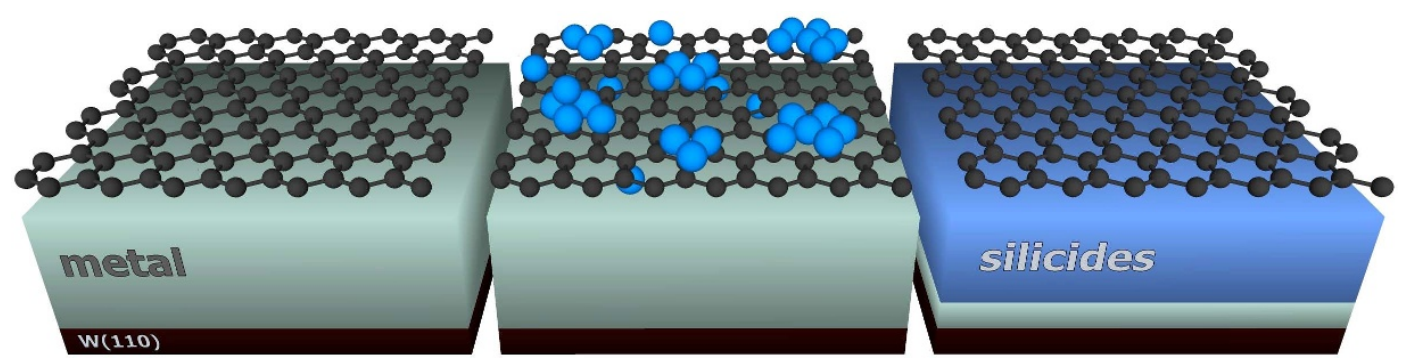

Figure 1 Schematic illustration of the proposed approach. At first, graphene is produced by CVD on $10 \mathrm{~nm}$-thick metallic films of Ni(111), Co(0001) or $\mathrm{Fe}(110)$ grown epitaxially on $\mathrm{W}(110)$. Then, cycles of silicon deposition and annealing are performed to promote intercalation of silicon atoms underneath graphene and initiate silicide formation. Desirable composition and properties of graphene-protected silicides depend on the amount of deposited silicon as well as temperature and duration of annealing.

\section{Results}

Fig. 1 schematically illustrates the proposed approach. In the beginning, a high-quality and large-area graphene layer is CVD-grown on a single-crystalline thin $\mathrm{Ni}$, Co or Fe film substrate. Then, silicon atoms are gradually deposited on top of the freshly prepared graphene layer. Their penetration underneath graphene occurs already at room temperature (RT). The mechanism of such intercalation process is based on the well-known fact that graphene grown on transition metal surfaces like $\mathrm{Ni}, \mathrm{Co}, \mathrm{Pt}$, Ru etc. possesses a domain structure. Thus, the intercalation of different elements like $\mathrm{Si}$ or $\mathrm{Au}$ occurs via the domain boundaries ${ }^{38}$. Since the solubility of $\mathrm{Si}$ in $\mathrm{Ni}$ is relatively high and the diffusion barrier for the deposited atoms on graphene is evidently quite low, silicon atoms will migrate to these domain boundaries and penetrate underneath graphene already at room temperature. In order to promote the chemical reaction between $\mathrm{Si}$ and metal, and obtain the desirable silicide phase, subsequent annealing cycles must be applied. The stoichiometry of the metal silicides can be controlled by the quantity of intercalated silicon as well as the duration and temperature of the annealing procedure. The silicon intercalation also considerably attenuates the strong hybridization between the electronic states of graphene and the substrate and, in particular cases, leads to the stabilization of the properties of quasi-freestanding graphene.

Graphene on Ni-silicide structures. We first go through the detailed analyses of the steps towards silicide formation in the case of $\mathrm{Ni}$.

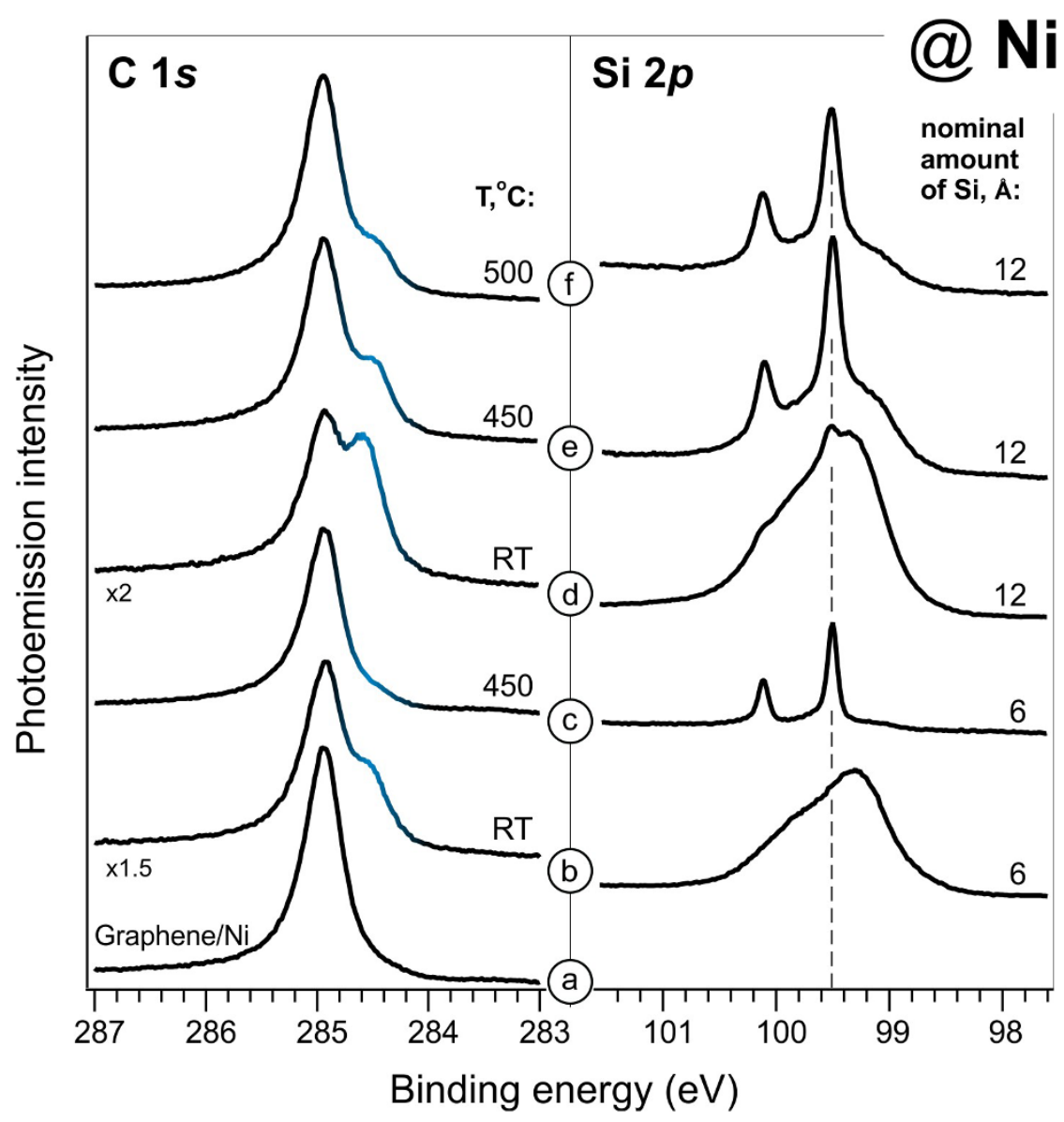

Figure $2 \mid$ Revealing the early stages of Si intercalation and silicide initiation on Ni substrate. The sequence of C $1 s$ and Si $2 p$ core level spectra for graphene/ $\mathrm{Ni}(111)$ (a), and with increasing doses of Si deposited on top, accompanied by annealing treatments (b-f). The highlighted part of the $\mathrm{C} 1 s$ spectra is associated with decoupled graphene regions. $1 \AA$ corresponds to the Si coverage of $2.88 \cdot 10^{14} \mathrm{at} / \mathrm{cm}^{2}$. The C $1 \mathrm{~s}$ and Si $2 p$ spectra were taken with $470 \mathrm{eV}$ and $135 \mathrm{eV}$ photons, respectively. 
Fig. 2 shows the core-level photo electron (PE) spectra of graphene freshly prepared on $\mathrm{Ni}(111)$, after deposition of small doses of silicon atoms, and after the post-annealing treatment. The $\mathrm{C} 1 \mathrm{~s}$ spectrum of native graphene/Ni at the bottom of the left panel looks very similar to that previously reported for this system ${ }^{13}$. Even a small dose $(\sim 6$ $\AA$ ) of silicon deposited at RT notably changes the spectral shape and gives rise to a shoulder at lower binding energies (BE). The Si $2 p$ spectrum does not reveal a well-resolved spin-orbit doublet. Instead, we see a rather extended and unshaped bump feature at the BE of $\sim 99.3 \mathrm{eV}$, which is consistent with the reported values for $\mathrm{Si} 2 p_{3 / 2}$ of clean silicon (which is $98.9-99.6 \mathrm{eV}$ depending on the surface reconstruction type). Together with a blurred LEED pattern (not shown) in comparison to that obtained from native graphene on nickel, these observations suggest that the major amount of the deposited silicon atoms creates disordered, amorphous islands on top. However, the shoulder in the C $1 s$ spectrum at $284.5 \mathrm{eV}$ implies that the penetration of silicon underneath graphene already starts at RT, and graphene is already partly lifted up and detached from the substrate ${ }^{39}$.

Annealing of this structure at $450^{\circ} \mathrm{C}$ for 40 minutes almost restores the shape of the $\mathrm{C} 1 s$ spectrum. At the same time, it leads to complete disappearance of the bump feature and the formation of a wellresolved Si $2 p$ spin-orbit doublet. The $\mathrm{BE}$ of the $\mathrm{Si} 2 p_{3 / 2}$ component is $99.5 \mathrm{eV}$, which is in a good agreement with the known values for nickel silicides ${ }^{40}$. Compared to the spectrum before annealing the Si $2 p$ intensity became notably smaller and the LEED pattern again reveals a sharp $(1 \times 1)$ hexagonal structure with low background.
Hence, the post-annealed state may be described as complete intercalation of silicon atoms underneath graphene. One may assume that due to their notable diffusion and relatively high solubility in nickel, the majority of Si atoms penetrate deep into the bulk of the substrate, thus becoming undetectable by XPS. This could reasonably explain the drop in the PE intensity of the Si $2 p$ spectrum. The restored shape of the $\mathrm{C} 1 \mathrm{~s}$ spectrum suggests that the chemical interaction of graphene with the underlying silicon-containing nickel substrate is similar to what was found for the native graphene/nickel system. Essentially, the intercalation of silicon atoms under graphene and their diffusion deeper into the $\mathrm{Ni}$ film leads to the formation of a NiSi solid solution in the subsurface region with a Si concentration up to the solubility limit of about 10 at. $\%^{41}$. Further increase of the $\mathrm{Si}$ concentration should give rise to Ni silicide formation starting from the $\mathrm{Ni}_{3} \mathrm{Si}$ phase. Thus, the $\mathrm{Ni}$ silicide formation should be steered by the balance of the intercalation rate and the bulk diffusivity.

Upon depositing further portions of silicon at RT and subsequent post-annealing processes, the system strictly follows the scenario described above. The Si $2 p$ spectrum taken immediately after the Si deposition reveals again a bump-like structure showing that plenty of amorphous silicon islands are formed on top of the graphene layer. Its PE intensity decreases considerably after the annealing cycles, and gradually, the well-resolved $\mathrm{Si} 2 p$ doublet becomes dominant. The latter, apparently, originates from the ordered phases of intercalated $\mathrm{Si}$ atoms in nickel. This time longer annealing at higher temperature, $500^{\circ} \mathrm{C}$, does not completely remove the bump in the Si $2 p$ spectrum. This may be explained by the formation of oxidized silicon islands

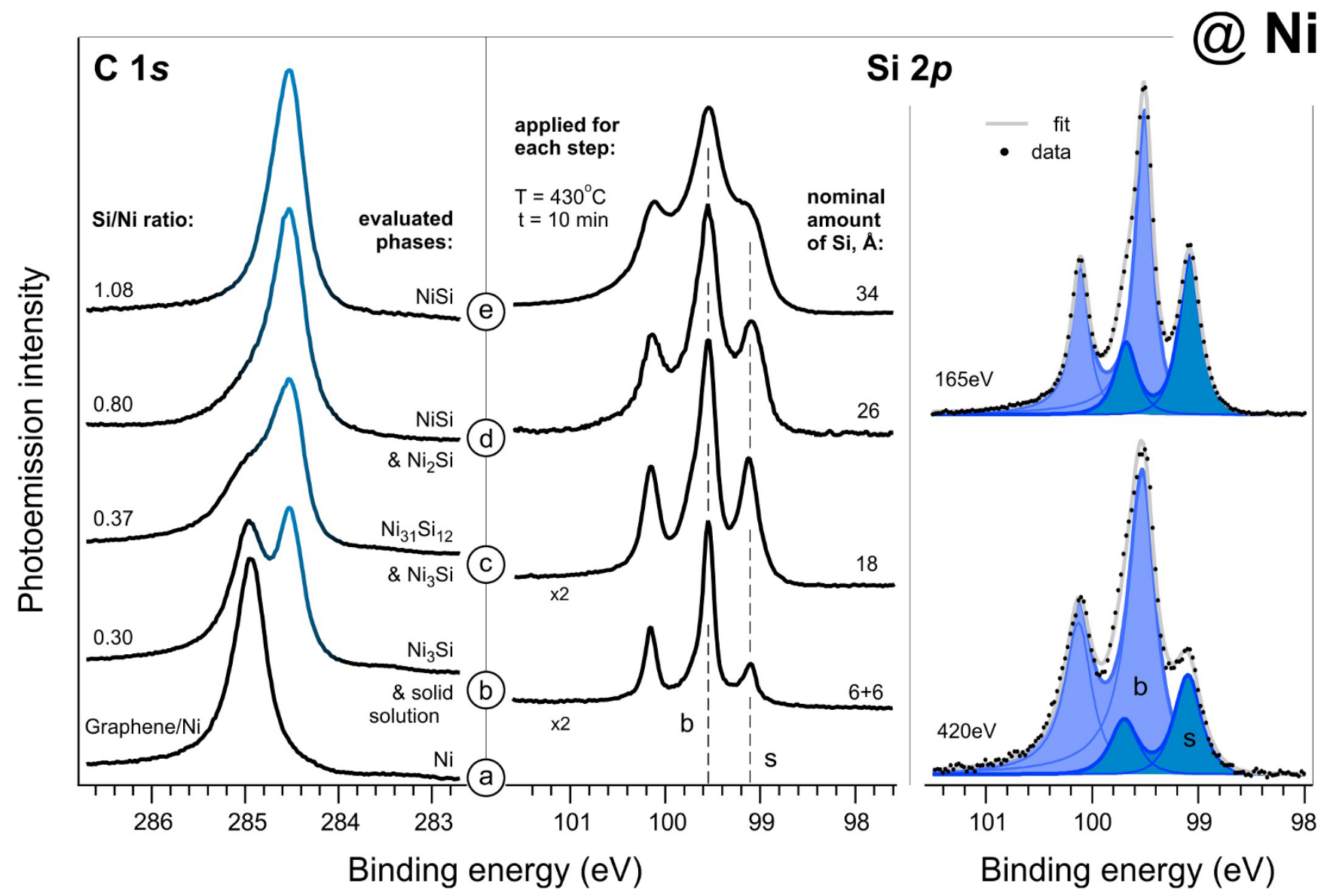

Figure 3 Monitoring the variety of graphene-capped Ni-silicide phases. Left panel: the sequence of C $1 s$ and Si $2 p$ spectra for freshly prepared graphene/ $\mathrm{Ni}(111)$ (a), and after gradual intercalation of Si promoted by annealing at $430^{\circ} \mathrm{C}$ for $10 \mathrm{~min}$ for each step (b-e). The highlighted part of the C $1 \mathrm{~s}$ spectra corresponds to decoupled graphene regions. Right panel: deconvolution of the Si $2 p$ spectrum (c) recorded with $165 \mathrm{eV}$ and $420 \mathrm{eV}$ photons. The surface (s) and bulk (b) components are highlighted. Si/Ni ratio is estimated from Si $2 p$ and Ni $3 p$ spectra and used for evaluation of the silicide stoichiometry. 


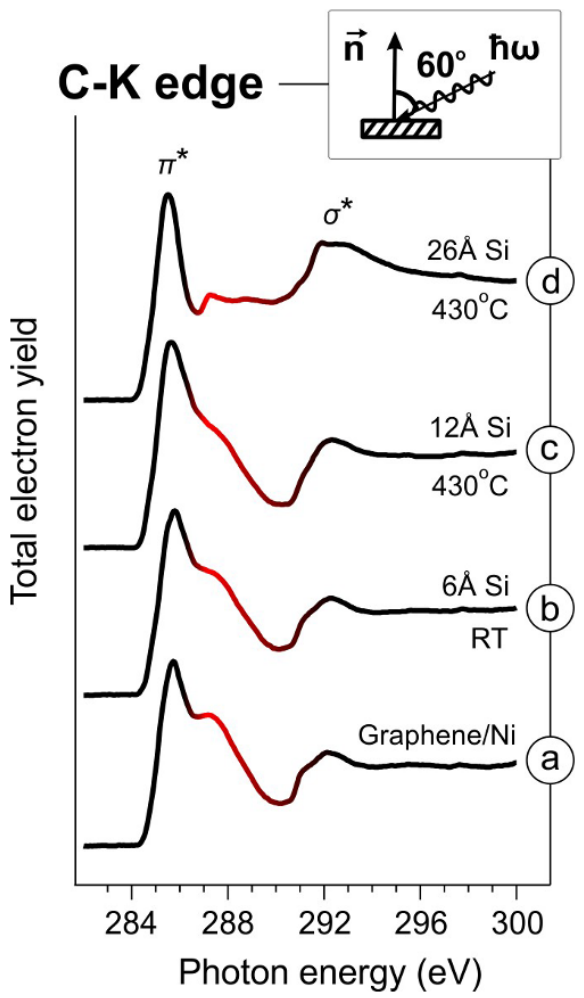

Figure 4 Evidence of electronic graphene-substrate decoupling upon silicides formation. NEXAFS spectra at the carbon K-edge for graphene/ $\mathrm{Ni}(111)$ (a), and for the systems with intercalated Si (b-d). The part of the spectrum that does not exhibit angular dependence is highlighted in red. The geometry of the experiment is depicted in the inset.

which were not intercalated under the given conditions. Clearly, the substrate becomes richer in silicon, implying the formation of the silicide phases underneath graphene. The LEED picture taken after the last treatment, corresponding to the upper PE spectra in Fig. 2, now reveals a sharp $(2 \times 2)$ structure with respect to the $\mathrm{Ni}(111)$ surface. The stoichiometry of the formed silicide will be discussed below.

The evolution of the system with increasing deposited, and subsequently intercalated silicon, up to $34 \AA$ of nominal thickness, is shown in Fig. 3. The series was measured on a freshly prepared graphene/nickel substrate. Each annealing step was done for $10 \mathrm{~min}$ and at a temperature of $430^{\circ} \mathrm{C}$.

This time, the shoulder at the lower $\mathrm{BE}$ in the $\mathrm{C} 1 s$ spectrum shaped into a well-defined peak at $284.5 \mathrm{eV}$. It is worth noting that we do not detect any sign of a PE signal at $\sim 283 \mathrm{eV}$ BE which would be indicative of carbide phases ${ }^{13}$, i.e. degradation of the graphene layer. It is interesting to compare the $\mathrm{C} 1 s$ spectra depicted in Fig. 2e and Fig. 3b, as the amount of deposited silicon in these two experiments was the same but the annealing temperatures are different. Apparently, reducing the temperature as well as the duration of annealing effectively increased the amount of silicon in the subsurface region underneath graphene. This is reflected in the $\mathrm{C} 1 \mathrm{~s}$ spectrum as a noticeable enhancement of the PE intensity at $284.5 \mathrm{eV}$. The LEED picture again reveals a sharp $(2 \times 2)$ pattern. Taking into account the silicon concentration estimated from the Si $2 p / \mathrm{Ni} 3 p$ intensity ratio we suppose, that such structure corresponds to the formation of ordered $\mathrm{Ni}_{3} \mathrm{Si}$ silicide under graphene. The solid state diffusion and the formation of a bulk alloy rather than a surface compound is supported by the fact that $\mathrm{Si}$ concentration decreases after annealing. This process is quite similar to the recently observed formation of the bulk $\mathrm{Ni}_{3} \mathrm{Al}$ phase with a $(2 \times 2)$ surface structure as a result of graphene intercalation with $\mathrm{Al}$ atoms ${ }^{42,43}$. The possible

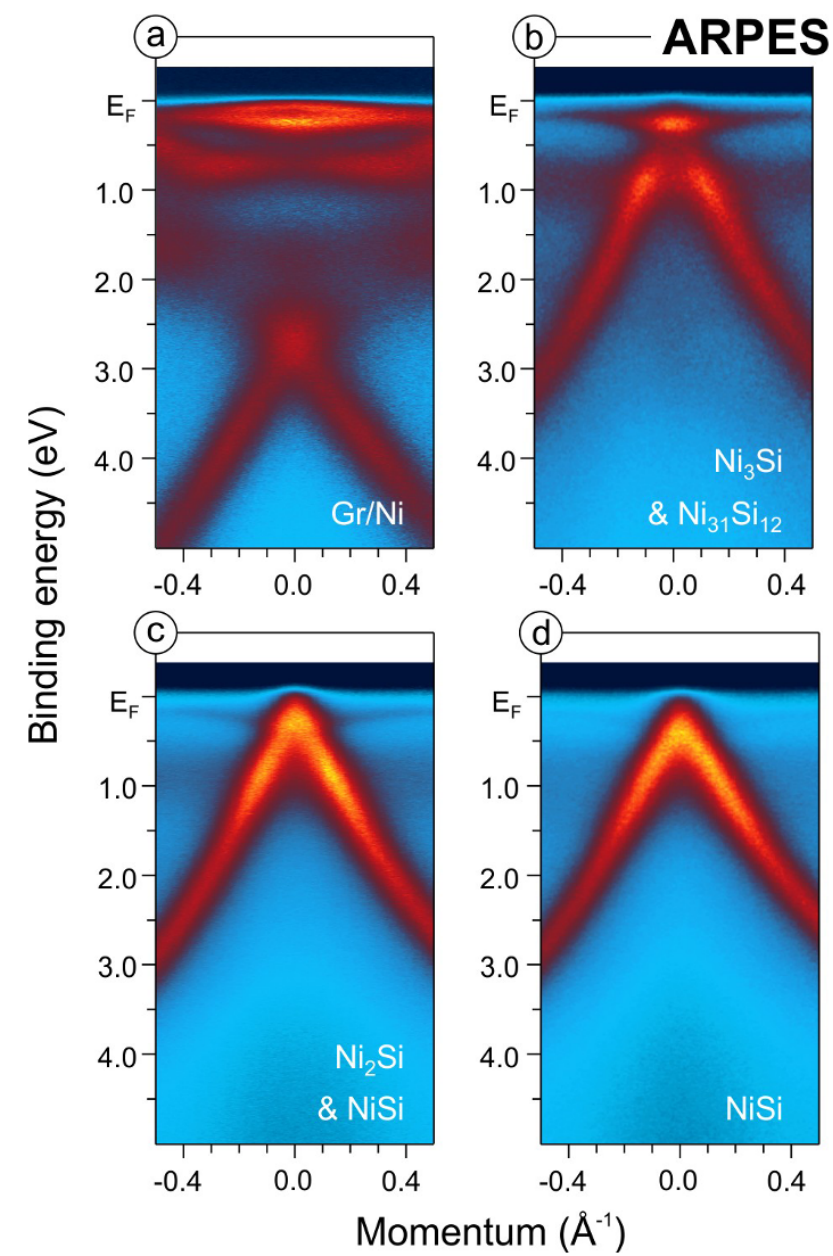

Figure $5 \mid$ Quasi-freestanding character of graphene on silicides. The sequence of ARPES data for graphene on $\mathrm{Ni}(111)$ (a), and at its detachment upon $\mathrm{Si}$ intercalation and silicide formation $(\mathrm{b}-\mathrm{d})$. The data were obtained near the K-point of graphene Brillouin zone using $40 \mathrm{eV}$ photons.

structural model is shown in Fig. S1. The Si $2 p$ spectral shape becomes very different from that of the Si-depleted systems described above. We see now a new well-defined peak at lower BE. To gain insight into the spectral structure of the discussed Si $2 p$ core-level spectrum, we recorded it at two different photon energies, $165 \mathrm{eV}$ and $420 \mathrm{eV}$, and then deconvolved the obtained Si $2 p$ spectra as shown in the right panel of the Fig. 3.

Both PE spectra were normalized to the maximum intensity. Each spectrum can be fitted with two doublets, where the two components likely reflect electron emission from $\mathrm{Si}$ atoms in the subsurface and in the bulk. The lower BE component is enhanced at the lower photon energy, which allows it to be assigned to the surface structure. Hence, the higher BE component originates from silicon atoms embedded in the bulk of the system.

We analyzed the $\mathrm{Si} 2 p / \mathrm{Ni} 3 p$ intensity ratio at each stage in order to evaluate the stoichiometry of the formed structure and to follow its evolution during the experiment. The obtained data together with the well-known phase diagrams for the $\mathrm{Ni}$-Si system suggest that starting from the solid solution we have passed from the Ni-rich phase $\mathrm{Ni}_{3} \mathrm{Si}$ to $\mathrm{NiSi}$. We have to admit that there may be a certain gradient of the silicon concentration from the surface to the bulk. Thus, for each particular system we may have several Ni-silicide phases $^{44}$ with higher Si concentration at the near-surface region, which is probed with XPS and solely defines the properties of the graphene-silicide interface. It should be noted that at low dosage of the intercalated silicon the multicomponent structure of the $\mathrm{C} 1 \mathrm{~s}$ 


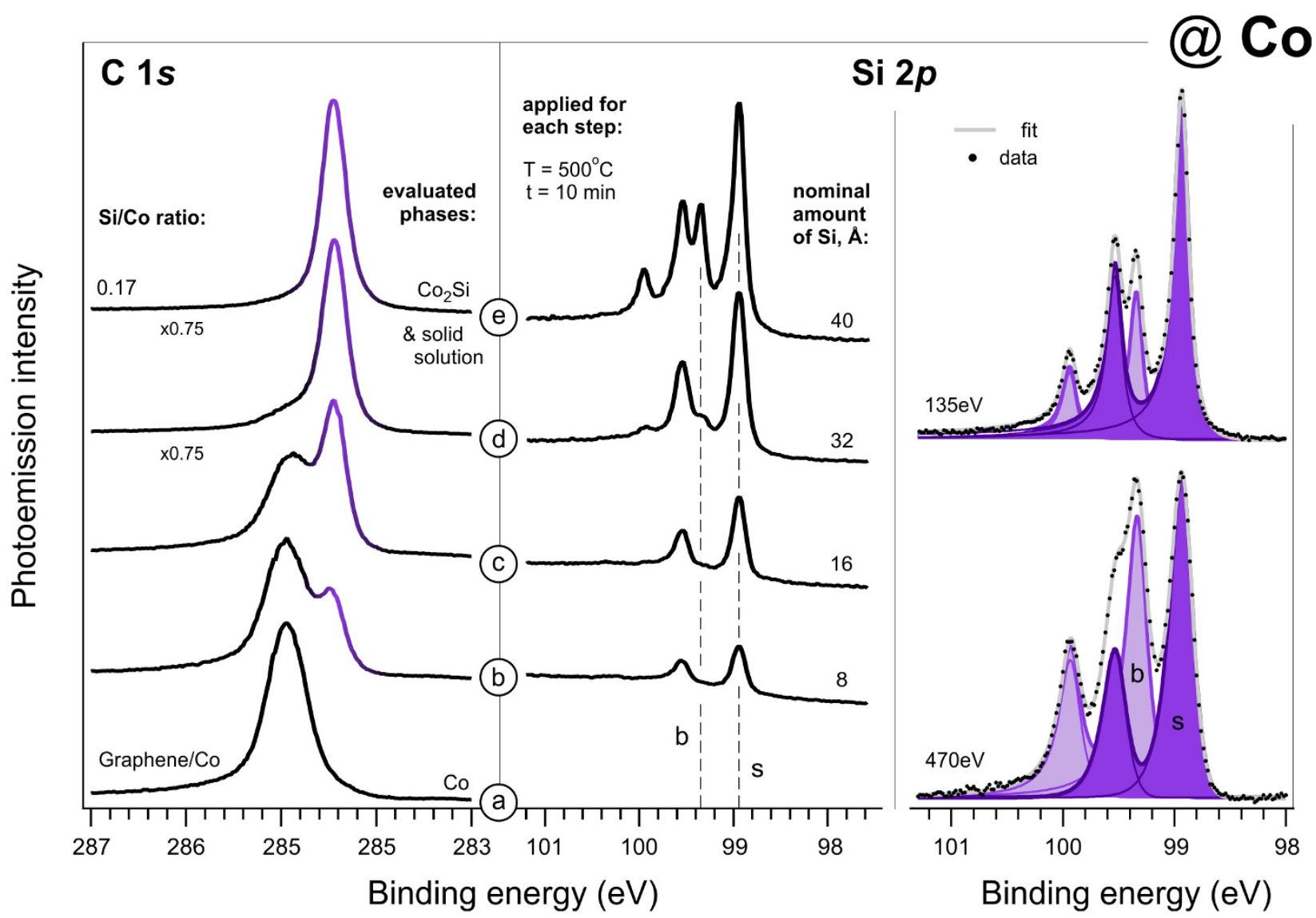

Figure 6 Formation of graphene-capped silicides in the Co-based system monitored with XPS. Evolution of the C $1 s$ and Si $2 p$ spectra with Si intercalation underneath graphene on $\mathrm{Co}(0001)$ (left panel). The highlighted part of the $\mathrm{C} 1 \mathrm{~s}$ spectra corresponds to decoupled graphene regions. Separation of the silicon atoms embedded in the cobalt layer near the surface and in the bulk obtained via deconvolution of the Si $2 p$ spectrum (right panel).

spectrum indicates coexistence of the graphene/silicide and graphene/nickel interfaces.

In Fig. 4, we show the evolution of NEXAFS C-K edge spectra, which allows us to follow possible modifications of the $\pi$ and $\sigma$ electronic states of graphene with increasing intercalated $\mathrm{Si}$. The shape of the $\mathrm{C}-\mathrm{K}$ edge spectrum of freshly prepared graphene on $\mathrm{Ni}(111)$ has been intensively discussed recently ${ }^{45}$. It contains two characteristic features, a double peak at $\sim 285 \mathrm{eV}$ and a manifold structure at $\sim 292 \mathrm{eV}$. They reflect C $1 s$ core electron excitations into the $\pi^{*}$ and $\sigma^{*}$ states, respectively. Both features are substantially broadened compared to what is observed for quasi-freestanding graphene because of the strong hybridization between the electron states of carbon and nickel. The red-colored parts of the spectra do not show the strong angular dependence typical for the $\pi^{*}$ and $\sigma^{*}$ features. This suggests that the $\mathrm{sp}^{2}$ character of the $\pi$ states is altered due to the interaction with the $\mathrm{Ni}$ atoms below. Upon the transition from the solid solution via $\mathrm{Ni}$-rich phases to the NiSi silicide, the lineshape of the spectrum becomes more and more similar to that of the freestanding graphene. This suggests that with increasing amount of silicon atoms inside nickel, the $\mathrm{Ni} 3 d$ states participate more actively in the formation of the Ni-Si bonds in silicides ${ }^{46}$ and, in turn, interact less and less with graphene. As a result, the electronic coupling between the graphene and the silicide substrate becomes weak.

This is clearly seen in Fig. 5, where we show the changes in the electronic band structure of graphene/Ni(111) with increasing intercalated $\mathrm{Si}$. The band structure for the freshly prepared graphene/Ni(111) is already well-known and has been a subject of extended discussions ${ }^{47-49}$. Due to the strong chemical interaction with the substrate, the $\pi$ band of graphene is strongly shifted in energy. The Dirac-point lies about $2.6 \mathrm{eV}$ below the Fermi level. However, even a small amount of intercalated $\mathrm{Si}$, corresponding to the $\mathrm{Ni}$-rich silicides, strongly changes the topology of the electron bands. The $\pi$ band is shifted back to lower BE again, and the cone apex appears quite close to the Fermi level. At the same time, the PE intensity of the respective $\mathrm{Ni} 3 d$ states considerably attenuates. With further increase of the intercalated Si and stabilization of silicide phases, the Diracpoint reaches the Fermi level, i.e. its position in the freestanding graphene.

Graphene on Co- and Fe-silicide structures. Having established a clear picture of the formation of $\mathrm{Ni}$-silicides under CVD-grown graphene, let us now turn to a brief description regarding $\mathrm{Co}-$ and Fe- based systems.

We performed the experiments in the same way and deposited first the silicon atoms on top of the freshly prepared graphene layer on monocrystalline films of cobalt or iron at RT. Later on, the annealing cycles were applied and the full process was monitored by analyzing the $C 1 s$ and Si $2 p$ spectra, which are depicted in Fig. 6 and Fig. 7, respectively. The stoichiometry of the metal-Si systems was evaluated via the $\mathrm{Si} 2 p / \mathrm{Co}(\mathrm{Fe}) 3 p$ ratio.

\section{Discussion}

Looking closely at the presented spectra, we may see and discuss certain similarities as well as differences of the silicide formation in these three systems. We should note that the silicide formation is solely defined by the local subsurface Si concentration, which, in turn, is governed by the balance of intercalation and diffusion rates. The local concentration of silicon atoms should be higher than that defined by the silicide stoichiometry, for example, not less than 50 at.\% for NiSi. Both diffusion and intercalation rates increase with 


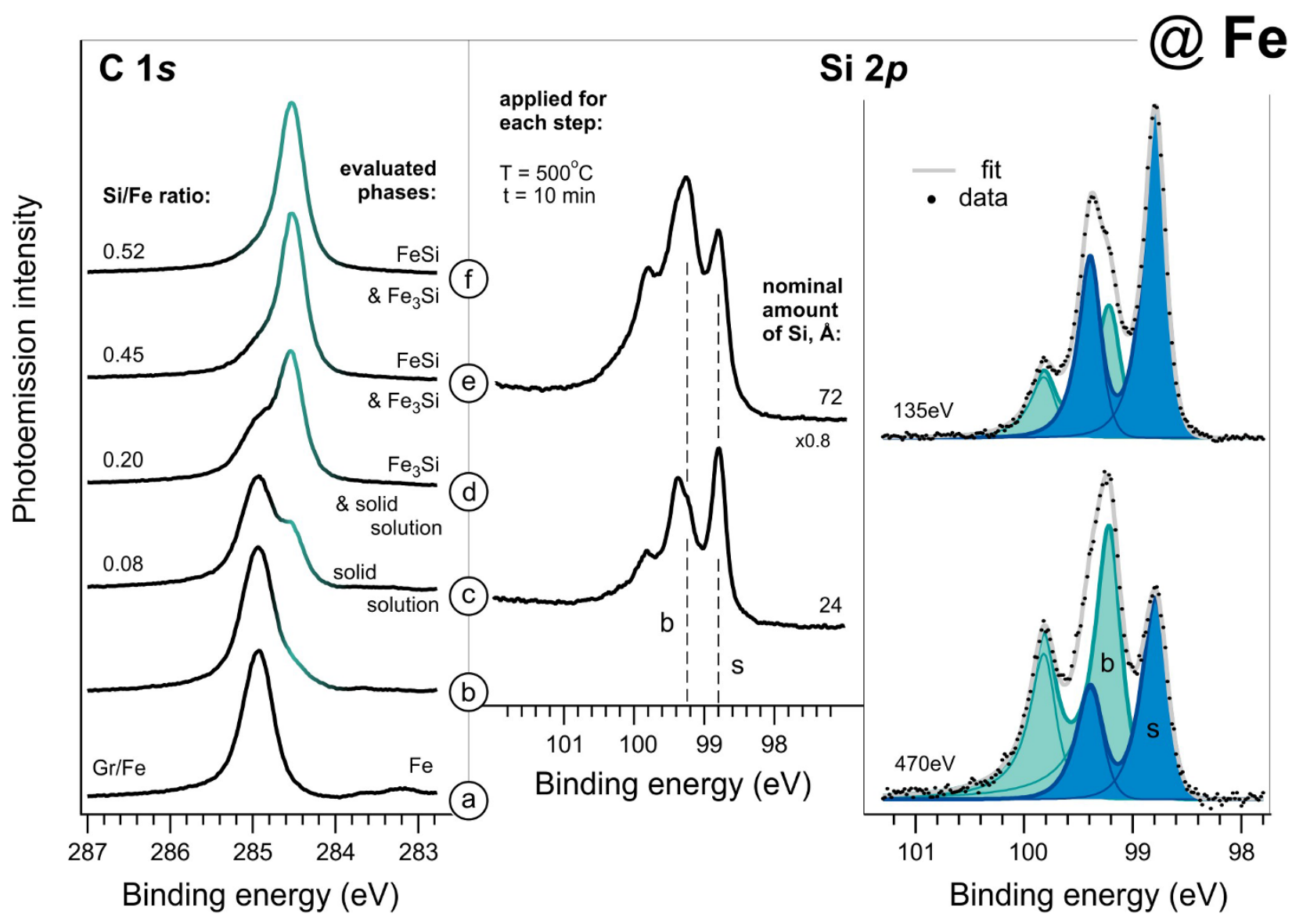

Figure $7 \mid$ Formation of graphene-capped silicides in the Fe-based system monitored with XPS. Evolution of the $\mathrm{C} 1 s$ and $\mathrm{Si} 2 p$ spectra with $\mathrm{Si}$ intercalation underneath graphene on a $\mathrm{Fe}(110)$ layer (left panel). The highlighted part of the $\mathrm{C} 1 s$ spectra corresponds to decoupled graphene regions. Separation of the silicon atoms embedded in the iron layer near the surface and in the bulk obtained via deconvolution of the Si $2 p$ spectrum (right panel).

temperature, these dependencies are described by Arrhenius equations with two different activation energies. The choice of the heat treatment temperature and duration can be performed using these equations. Unfortunately, the lack of fundamental information on this matter allows us to explain the behavior of these three systems on the phenomenological level only.

Despite the fact that $\mathrm{Ni}$ and Co metals exhibit very similar properties, the difference in the behavior of graphene/ $\mathrm{Ni}$ and graphene/Co systems upon $\mathrm{Si}$ intercalation can be explained as follows: the $\mathrm{Ni}_{3} \mathrm{Si}$ phase exists in a wide temperature range in the $\mathrm{Ni}$-rich part of the $\mathrm{Ni}$ $\mathrm{Si}$ system. In contrast, the $\mathrm{Co}_{3} \mathrm{Si}$ phase is observed only within a short range of relatively high temperatures. The $\mathrm{Ni}_{3} \mathrm{Si}$ phase has a similar crystal structure to $\mathrm{Ni}$, i.e. it arises due to the ordering in $\mathrm{Ni}$-Si solid solution. In such systems typically the interactions between different atoms are stronger than for the atoms of the same element. This enhanced interaction, in turn, promotes interdiffusion due to thermodynamic reasons $s^{50}$. Because of this, the Si diffusion in Ni is notably faster than that in Co in the corresponding metal-rich solid solutions. Therefore, in the Co-based systems the surface-related component of the Si $2 p$ spectrum always has higher intensity than the bulk one, which is not the case for the Ni-based structures. Note that for Fesystems the situation becomes somewhat intermediate, because similar to $\mathrm{Ni}, \mathrm{Fe}$ forms extended solid solutions with $\mathrm{Si}$ up to the ordered intermetallic phases $\mathrm{Fe}_{3} \mathrm{Si}$. Hence, the discussion given above is true for the graphene on iron system, too. However, in contrast to nickel, iron has a higher melting point. Therefore, the bulk diffusion is activated at essentially higher temperature. Thus, at the same temperature, Si evidently has higher diffusivity in nickel crystal than in iron one. This is in line with our spectral observations described above.
In summary, we have demonstrated that the ordered silicides of $\mathrm{Ni}, \mathrm{Co}$ and $\mathrm{Fe}$ with different and well-defined stoichiometries can be produced under CVD-grown graphene. The obtained materials are reliably protected by the graphene layer, making them compatible for a variety of nanoscaled applications at ambient conditions. Our finding suggests that the graphene layer is weakly electronically coupled to the silicide phases, thus keeping its remarkable properties ready for use. We anticipate that the presented results will create a solid platform to further explore the opportunity to integrate graphene with the mature silicon-based technology. We believe that this would give rise for further exploitation of the unique properties of different metal silicides under graphene's umbrella as well as the properties of graphene itself for the widely discussed applications in the nextgeneration electronic devices.

\section{Methods}

The studied graphene samples were synthesized in situ by CVD from propylene $\left(\mathrm{C}_{3} \mathrm{H}_{6}\right)$ on the $\mathrm{Ni}(111), \mathrm{Co}(0001)$ and $\mathrm{Fe}(110)$ surfaces. For this purpose we prepared metallic films with a thickness of $\sim 10 \mathrm{~nm}$ grown epitaxially on the $\mathrm{W}(110)$ substrate in ultra-high vacuum conditions. The high crystalline quality of the studied samples was confirmed by the sharp low energy electron diffraction (LEED) patterns. After that, graphene layer was grown on nickel and cobalt surfaces at a pressure of propylene of $\sim 1 \cdot 10^{-6} \mathrm{mbar}$ and the substrate temperature of $600^{\circ} \mathrm{C}$ during $15 \mathrm{~min}$. At these conditions the reaction is well-known to be self-limited to single graphene layer. The parameters of CVD for the iron-based system were slightly increased to avoid carbidization and produce a complete graphene layer, namely $5 \cdot 10^{-6} \mathrm{mbar}, 700^{\circ} \mathrm{C}$ and $25 \mathrm{~min}$, respectively (see Supplementary Note 1 for details). The penetration of the silicon atoms into the metal substrates and the subsequent formation of various types of silicides underneath the graphene top layer were studied using X-ray photoelectron spectroscopy (XPS), angle resolved photoelectron spectroscopy (ARPES), near-edge X-ray absorption fine structure spectroscopy (NEXAFS), and LEED. The well-known stability of graphene-based systems to ambient atmospheric 
conditions $s^{51-53}$ was proved for the studied structures, too. Experiments were performed at BESSY synchrotron radiation facility - at the beamlines U125/2-SGM, UE56/2-PGM-2 and at the Russian-German beamline. ARPES measurements were performed at room temperature with a Scienta R4000 spectrometer.

1. Novoselov, K. S. et al. A roadmap for graphene. Nature 490, 192 (2012).

2. Levendorf, M. P., Ruiz-Vargas, C. S., Garg, S. \& Park, J. Transfer-free batch fabrication of single layer graphene transistors. Nano Lett. 9, 4479 (2009).

3. Kim, K. S. et al. Large-scale pattern growth of graphene films for stretchable transparent electrodes. Nature 457, 706 (2009).

4. Hofrichter, J. et al. Synthesis of graphene on silicon dioxide by a solid carbon source. Nano Lett. 10, 36 (2010).

5. Wessely, P. J., Wessely, F., Birinci, E., Schwalke, U. \& Riedinger, B. Transfer-free fabrication of graphene transistors. J. Vac. Sci. Technol. B 30, 03D114 (2012).

6. Zenasni, A., Delamoreanu, A. \& Rabot, C. Free-suspended graphene synthesis via carbon diffusion through platinum-based metal. Appl. Phys. Lett. 100, 151907 (2012).

7. Meng, L. et al. Silicon intercalation at the interface of graphene and $\operatorname{Ir}(111)$. Appl. Phys. Lett. 100, 083101 (2012).

8. Mao, J. et al. Silicon layer intercalation of centimeter-scale, epitaxially grown monolayer graphene on $\mathrm{Ru}(0001)$. Appl. Phys. Lett. 100, 093101 (2012).

9. Cui, Y. et al. An exchange intercalation mechanism for the formation of a twodimensional Si structure underneath graphene. Nano Res. 5, 352 (2012).

10. Lizzit, S. et al. Transfer-free electrical insulation of epitaxial graphene from its metal substrate. Nano Lett. 12, 4503 (2012).

11. Eizenberg, M. \& Blakely, J. M. Carbon monolayer phase condensation on $\mathrm{Ni}(111)$. Surf. Sci. 82, 228 (1979).

12. Reina, A. et al. Large area, few-layer graphene films on arbitrary substrates by chemical vapor deposition. Nano Lett. 9, 30 (2009).

13. Grüneis, A., Kummer, K. \& Vyalikh, D. V. Dynamics of graphene growth on metal surface: a time-dependent photoemission study. New J. Phys. 11, 073050 (2009).

14. Varykhalov, A. \& Rader, O. Graphene grown on $\mathrm{Co}(0001)$ films and islands: Electronic structure and its precise magnetization dependence. Phys. Rev. B 80 035437 (2009).

15. Vinogradov, N. A. et al. Formation and structure of graphene waves on $\mathrm{Fe}(110)$. Phys. Rev. Lett. 109, 026101 (2012).

16. Lin, Y.-C., Chen, Y. \& Huang, Y. The growth and applications of silicides for nanoscale devices. Nanoscale 4, 1412 (2012).

17. Schmitt, A. L., Higgins, J. M., Szczech, J. R. \& Jin, S. Synthesis and applications of metal silicide nanowires. J. Mater. Chem. 20, 223 (2010).

18. Lavoie, C., d'Heurle, F. M., Detavernier, C. \& Cabral Jr., C. Towards implementation of a nickel silicide process for CMOS technologies. Microelectron. Eng. 70, 44 (2003)

19. Kittl, J. A. et al. Silicides and germanides for nano-CMOS applications. Mater. Sci. Eng. B 154, 144 (2008).

20. Bhaskaran, M., Sriram, S. \& Sim, L. W. Nickel silicide thin films as masking and structural layers for silicon bulk micro-machining by potassium hydroxide wet etching. J. Micromech. Microeng. 18, 095002 (2008).

21. Senthilarasu, S., Sathyamoorthy, R. \& Lalitha, S. Synthesis and characterization of $\beta-\mathrm{FeSi}_{2}$ grown by thermal annealing of $\mathrm{Fe} / \mathrm{Si}$ bilayers for photovoltaic applications. Sol. Energ. Mat. Sol. C. 82, 299 (2004).

22. Kim, J. \& Anderson, W. A. Metal silicide-mediated microcrystalline silicon thinfilm growth for photovoltaics. Sol. Energ. Mat. Sol. C. 91, 534 (2007).

23. Zhou, F. et al. Determination of transport properties in chromium disilicide nanowires via combined thermoelectric and structural characterizations. Nano Lett. 7, 1649 (2007).

24. Chen, X., Wang, X., Xiu, J., Williams, C. T. \& Liang, C. Synthesis and characterization of ferromagnetic nickel-cobalt silicide catalysts with good sulfur tolerance in hydrodesulfurization of dibenzothiophene. J. Phys. Chem. C 116, 24968 (2012).

25. Ko, F.-H., Yeh, Z.-H., Chen, C.-C. \& Liu, T.-F. Self-aligned platinum-silicide nanowires for biomolecule sensing. J. Vac. Sci. Technol. B 23, 3000 (2005).

26. Kittl, J. et al. CMOS integration of dual work function phase-controlled Ni fully silicided gates (NMOS: NiSi, PMOS: $\mathrm{Ni}_{2} \mathrm{Si}$, and $\mathrm{Ni}_{31} \mathrm{Si}_{12}$ ) on HfSiON. IEEE, Electr. Device L. 27, 966 (2006).

27. Kittl, J. A. et al. Kinetics of $\mathrm{Ni}_{3} \mathrm{Si}_{2}$ formation in the $\mathrm{Ni}_{2} \mathrm{Si}-\mathrm{NiSi}$ thin film reaction from in situ measurements. Appl. Phys. Lett. 91, 232102 (2007).

28. Dasgupta, N. P. et al. Nickel silicide nanowire arrays for anti-reflective electrodes in photovoltaics. Adv. Funct. Mater. 22, 3650 (2012).

29. Fan, X., Zhang, H., Du, N. \& Yang, D. Phase-controlled synthesis of nickel silicide nanostructures. Mater. Res. Bull. 47, 3797 (2012).

30. Seo, K. et al. Magnetic properties of single-crystalline CoSi nanowires. Nano Lett. 7,1240 (2007)

31. Qu, Y. et al. Synthesis and electric properties of dicobalt silicide nanobelts. Chem. Commun. 11 47, 1255 (2011).
32. Girlanda, R., Piparo, E. \& Balzarotti, A. Band structure and electronic properties of $\mathrm{FeSi}$ and $\alpha-\mathrm{FeSi}_{2}$. J. Appl. Phys. 76, 2837 (1994).

33. Bost, M. C. \& Mahan, J. E. Optical properties of semiconducting iron disilicide thin films. J. Appl. Phys. 58, 2696 (1985).

34. Pronin, I. et al. Magnetic ordering of the Fe/Si interface and its initial formation. J. Appl. Phys. 104, 104914 (2008).

35. Izumi, S., Shaban, M., Promros, N., Nomoto, K. \& Yoshitake, T. Near-infrared photodetection of $\beta-\mathrm{FeSi}_{2} / \mathrm{Si}$ heterojunction photodiodes at low temperatures. Appl. Phys. Lett. 102, 032107 (2013).

36. Lenz, K. et al. Magnetic properties of $\mathrm{Fe}_{3} \mathrm{Si} / \mathrm{GaAs}(001)$ hybrid structures. Phys. Rev. B 72, 144411 (2005)

37. Hafner, J. \& Spišák, D. Structure and stability of the low-index surfaces of $\mathrm{Fe}_{3} \mathrm{Si}: A b$ initio density functional investigations. Phys. Rev. B 75, 195411 (2007).

38. Tontegode, A. Ya. and Rut'kov, E. V. Intercalation by atoms of a two-dimensional graphite film on a metal. Phys.-Usp. 36, 1053 (1993).

39. Usachov, D. et al. Nitrogen-doped graphene: Efficient growth, structure, and electronic properties. Nano Lett. 11, 5401 (2011).

40. Cao, Y., Nyborg, L. \& Jelvestam, U. XPS calibration study of thin-film nickel silicides. Surf. Interface Anal. 41, 471 (2009).

41. ASM handbook: Vol. 3: Alloys, Phase diagrams (ASM International, 1992).

42. Addou, R., Dahal, A. \& Batzill, M. Graphene on ordered Ni-alloy surfaces formed by metal (Sn, Al) intercalation between graphene/Ni(111). Surf. Sci. 606, 1108 (2012).

43. Rybkina, A. A. et al. Interaction of graphene with intercalated Al: The process of intercalation and specific features of the electronic structure of the system. Surf. Sci. 609, 7 (2013).

44. Gaudet, S., Desjardins, P. \& Lavoie, C. The thermally-induced reaction of thin Ni films with Si: Effect of the substrate orientation. J. Appl. Phys. 110, 113524 (2011).

45. Dedkov, Yu. S. \& Fonin, M. Electronic and magnetic properties of the grapheneferromagnet interface. New J. Phys. 12, 125004 (2010).

46. Rubloff, G. W. Microscopic properties and behavior of silicide interfaces. Surf. Sci. 132, 268 (1983)

47. Oshima, C. \& Nagashima, A. Ultra-thin epitaxial films of graphite and hexagonal boron nitride on solid surfaces. J. Phys.: Condens. Matt. 9, 1 (1997)

48. Grüneis, A. \& Vyalikh, D. V. Tunable hybridization between electronic states of graphene and a metal surface. Phys. Rev. B 77, 193401 (2008).

49. Varykhalov, A. et al. Intact Dirac cones at broken sublattice symmetry: Photoemission study of graphene on Ni and Co. Phys. Rev. X 2, 041017 (2012).

50. Murch, G. E. Diffusion kinetics in solids, in Phase Transformations in Materials (Wiley-VCH Verlag GmbH \& Co. KGaA, 2005) pp. 171-238.

51. Dedkov, Y. S., Fonin, M. \& Laubschat, C. A possible source of spin-polarized electrons: The inert graphene/Ni(111) system. Appl. Phys. Lett. 92, 052506 (2008).

52. Dedkov, Y. S., Fonin, M., Rüdiger, U. \& Laubschat, C. Graphene-protected iron layer on $\mathrm{Ni}(111)$. Appl. Phys. Lett. 93, 022509 (2008).

53. Marchenko, D., Varykhalov, A., Rybkin, A., Shikin, A. M. \& Rader, O. Atmospheric stability and doping protection of noble-metal intercalated graphene on Ni(111). Appl. Phys. Lett. 98, 122111 (2011).

\section{Acknowledgements}

This work was supported by BMBF (grant No. 05K12OD3), RFBR (No. 11-03-00752) and St. Petersburg State University (No. 11.37.634.2013) grants. D.U., O.V. and A.F. acknowledge funding from the President's grant MK-3303.2012.2. We acknowledge Helmholtz Zentrum Berlin für Materialien und Energie for support within bilateral Russian-German Laboratory program.

\section{Author contributions}

O.V., D.U. and D.V.V. designed the research. O.V., A.F., D.U., A.V.G., K.B. and A.G. have participated in XPS, NEXAFS and ARPES experiments. Operation of the XPS and ARPES facilities was carried out by O.V., A.F. and D.U. Theoretical modeling was performed by L.V.Y. and N.I.V. All authors discussed the results. The manuscript was written by O.V., L.V.Y., D.U. and D.V.V. All authors have read and approved the decisive version of the manuscript.

\section{Additional information}

Supplementary information accompanies this paper at http://www.nature.com/ scientificreports

Competing financial interests: The authors declare no competing financial interests.

How to cite this article: Vilkov, O. et al. Controlled assembly of graphene-capped nickel, cobalt and iron silicides. Sci. Rep. 3, 2168; DOI:10.1038/srep02168 (2013).

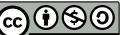

This work is licensed under a Creative Commons AttributionNonCommercial-ShareAlike 3.0 Unported license. To view a copy of this license, visit http://creativecommons.org/licenses/by-nc-sa/3.0 